CITATION: Connor, A.M. \& Siringoringo, W. (2017) "Evolutionary Optimisation to Minimise Material Waste in Construction”, International Journal of Computational Systems Engineering, 3(4), 183-192, DOI: 10.1504/IJCSYSE.2017.10010096

\section{Evolutionary Optimisation to Minimise Material Waste in Construction}

\section{Andy M. Connor \& Wilson Siringoringo}

\begin{abstract}
This paper describes the development and evaluation of a range of metaheuristic search algorithms applied to the optimal design of two-dimensional layout problems, with the particular application on residential building construction. Results are presented to allow the performance of the different algorithms to be compared in the pareto-optimal solution space, with resulting solutions identified and analysed in the objective space. These results show that all of the algorithms investigated have the potential to be applied to optimise material layout and improve the design processes used during building construction.
\end{abstract}

Keywords: Metaheuristic algorithms, Evolutionary computation, Layout optimisation, Residential construction

\section{Introduction}

The utilisation of computer-assisted processes in the civil construction industry results in ever increasingly rich sets of data being generated and stored in electronic formats. The availability of technical data, such as that of electronic drafting documents, offers the opportunity to integrate optimisation into the design, planning, and construction of the structure.

This paper describes the development and evaluation of a generic framework for multiobjective optimisation on the design that involves two-dimensional physical objects, with the particular application on residential building construction. The underlying premise of the research is that the value and performance of a building, in a limited number of measurable criteria, can be improved from the original design by altering the design choices as permitted by the available data. However, while the multiobjective optimisation framework in this research specifically targets building construction objects, the methods and techniques utilised are not specific to these problems and therefore can be applied to similar problems in different contexts beyond what is covered in this paper.

The optimisation of two-dimensional layout is a class of problems applicable to many industries. The problems call for the packing of non-overlapping shapes in an enclosed plane with the primary aim of minimizing the area outside the boundaries of the shapes, therefore maximizing the utilization of the material in the base sheet. Two-dimensional layout problem exists in several variants. To name a few there are the sheet layout problem, bin packing and strip packing problems, optimum floor plan problem, and cutting stock problem. However, none of the classically defined classes of layout optimisation embrace the potential for material reuse that is available in residential construction.

This paper is structured as follows. Section 2 provides and overview of the background to this work, whilst Section 3 provides and overview of layout optimisation problems common in residential construction. Section 4 describes a specific case problem for which results are presented in Section 5. These results and their implications are discussed in Section 5 and finally, Section 6 concludes the paper.

\section{Background \& Related Work}

The research described in this paper seeks to utilise metaheuristic algorithms to solve multi-objective layout optimisation problems inherent in the design and construction of residential structures. Prior research has been done in various fields of two-dimensional layout optimisation problem as driven by the practical needs from the industry. There majority of such problems can be modelled in one of the four main variants: the sheet layout, the bin packing and strip packing, rectangular, floor planning and cutting stock problem. With the sheet layout, bin packing and strip packing versions being the most relevant to this research.

Dyckhoff (1990) makes an attempt to provide a systematic classification of such optimisation problems. He uses the term cutting and packing ( $\mathrm{C} \& \mathrm{P}$ ) as a generic name for the problem and all its variants. He further postulates that there are four properties of each problem that determine to which class it belongs. The properties are the problem dimensionality, the kind of assignment, the assortment of the large objects (containers), and the assortment of the small items (pieces). The four properties are refined into finite set of sub-properties that relate to dimensionality, the type of assignment, the assortment of large objects and the assortment of small items (Dyckhoff, 1990).

Wascher, Heike, and Schumann (2007) later proposes an updated classification system that is largely based on Dyckhoff's model. In the new topology system, the "kind of assignment" and "assortment of the small items" are no longer evaluated as separate items, but combined to determine a new property basic problem type. Further, Dyckhoff's "assortment of the large objects" is added to determine a new property intermediate problem type. Finally, the "problem dimensionality" contributes to establish the refined problem type.

Wascher et al. (2007) however, agree with Dyckhoff (1990) that any variant of the C \& P problem are composed of five sub-problem that must be resolved in order to achieve optimum result:

\section{The problem of selecting of the large objects}


2. The problem of selecting of the small items

3. The problem of grouping the selected small items

4. The problem of assigning the subsets of the small items to the large objects

5. The layout problem regarding the arrangement of the small items on each of the selected large objects with respect to the geometric condition

The two classification schemes outlined above have been used to guide a search of the literature for specific instances of layout optimisation approaches that can be used to inform a suitable approach for residential construction where the goals are to not only minimise waste but to do so be re-using the offcuts in the optimisation approach. The work of Adamowicz and Albano (1976), who investigated the layout of steel sheets in ship building, bears some resemblance to the problems examined in this paper however the use of material off cuts is not specifically investigated. In contrast, Sibley-Punnet and Bossomaier (2001) investigate the reuse off cuts from corrugated iron roofs using the high cost of delivering the roofing material to justify the computational effort required. However, the representation of the problem is only partially abstracted from the constraints of the material (Cranny, Bossomaier, \& Sibley-Punnett, 1999) which limits the extent to which it can be generalised to a range of problems in residential construction. Sibley-Punnet and Bossomaier (2001) also utilised singly objective optimisation which is a limitation in terms of understanding the trade-offs between different possible design objectives.

Whilst the literature related to material reuse is limited, there is a broad range of applications related to layout optimisation in general. Epstein, Imreh, and Levin (2010) report that algorithms used to solve layout problems fall into three classes, namely approximation algorithms, lower bounds algorithms, and exact algorithms. An example of an approximation algorithm is provided by Vassiliadis (2005) who creates a model of a $2 \mathrm{D}$ bin packing problem using a binary tree data structure and local search optimisation methods. He argues that a tree-representation in his design is capable of capturing any configuration and translations of the problem efficiently and offers strong base for the optimisation algorithms that follow. He further specifies simulated annealing and threshold accepting to implement the local search.

Another example of a 2D bin packing approximation algorithm is provided by (Shigehiro, Koshiyama, \& Masuda, 2005), which is based on tabu search. In their algorithm, various close permutations of the rectangles formation are explored to find local optima while maintaining the list of previously known optimum solutions. Later Rao, Geevarghese, and Rajan (2010) propose another approach based on the combination of the Linear Programming relaxation and a greedy heuristic.
Solutions based on lower bounds algorithm also have been proposed over the years, as reported by Lodi, Martello, and Vigo (2004) and Harren and Kern (2011).

The characteristics of the exact algorithm is described by Labbé, Laporte, and Martello (1995). Exact algorithm solution for strip packing is proposed by Hifi (1998). First he decomposes the 2D strip packing problem into a series of two-dimensional constrained cutting stock problems, then branch-and-bound procedures are used to compute the final result. A more recent example is provided by Coté, Gendreau, and Potvin (2014) who uses branch-and-cut technique to solve the two-dimensional orthogonal packing problem.

Another important variant of the sheet nesting problem is the cutting stock problem, where a single stock sheet is to be cut into a series of rectangular pieces of predetermined sizes. The sizes are usually associated to values, from which the objective function of the optimisation is constructed. This type of problem is common in the iron and steel industries. Tokuyama and Ueno (1985) define that such application is characterised by varying criteria such as maximizing yield or increasing efficiency of production lines. The cutting stock problem is accompanied by an optimal stock selection problem, and as such bears some similarity to the issues encountered during

Similar to bin packing and strip packing, typically the stock material in the cutting stock problems has a rectangular shape. In some cases however, the material can have an irregular outline as well as defective spots in the internal area. Georgis, Petrou, and Kittler (2000) define the generalized CSP and propose a solution to such problem based on simulated annealing technique. Whilst this bears some similarity to the voids encountered in residential construction, there is still a need to fully define the problems encountered during layout optimisation in residential construction.

Such layout optimisation has been addressed to some degree in the existing literature. For example, Nimtawat, and Nanakorn (2010) investigate the combination of beams and slabs in the layout of reinforced concrete floors whereas Hong, Lee, Lee \& Kim (2014) consider the optimisation of structural elements. There is a growing body of work that examines building envelopes as an optimisation problem (Echenagucia, Capozzoli, Cascone, \& Sassone, 2015), including how the material usage can impact the energy efficiency of a building (Yang, Lin, Lin, \& Tsai, 2017). Whilst material layout has received little interest in recent studies, there is a pressing need to consider the sustainability of construction projects and hence material reuse can be considered as one element of sustainable building design (Wang \& Adeli, 2014) that is already under consideration in other industries (Snudden, Ward, \& Potter, 2014). The need for so-called "waste avoidance” (Crossin, Hedayati, \& Clune, 2014) in residential construction has been identified as a means to address the issue of building and construction waste 
materials continuing to be a major problem causing significant environmental impact (Zou, Hardy, \& Yang, 2015).

\section{Residential Construction Optimisation}

Successful application of optimisation on building designs requires a number of decisions made at various levels. For example, business stakeholders require the optimisation solution to yield appreciable return to their investment. Whereas from the technical perspective, the optimisation domain and scope need to be limited to those feasible with available technologies and computing resources. These requirements provide the grounds to the decision on which aspects of building design are to be optimised.

Flat surfaces make prime candidate for the application of layout optimisation on house design. A polygon shaped area such as wall, or, or ceiling is to be tiled with covering sheet material such as drywall or plywood. With such tiling, it is required that the entire surface is covered with no gaps or overlaps. The panels are obtained from the supplier in fixed size rectangular sheets. Typically the individual panel is much smaller than the area to be covered. It is also anticipated that the enclosing area may have an irregular outline. To keep the construction expenses under control, the builder must arrange the panels in a way that keeps the cost variables low. Such parameters include the number of panels allocated, the amount of discarded off cuts, and the amount of effort required for moving and cutting the panels.

The problem is demonstrated in Figure 1.
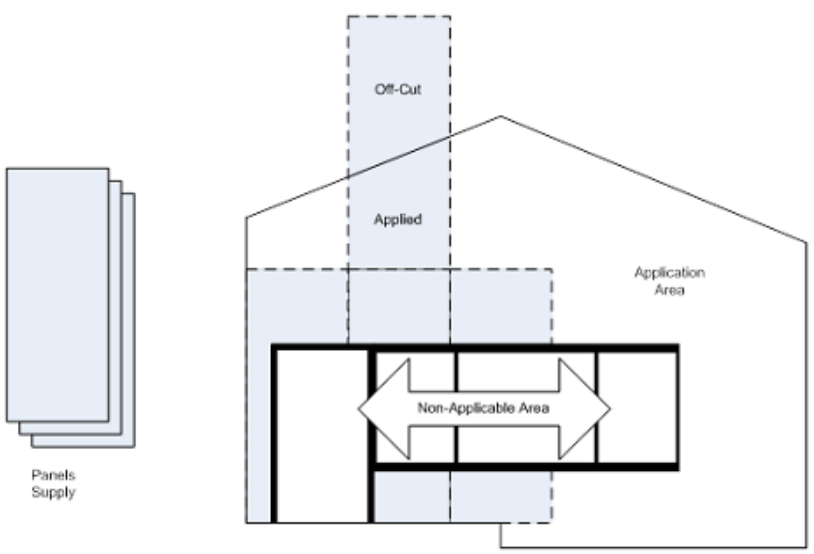

Figure 1. Tiling a wall with fixed size panels

This problem differs slightly from many layout optimisation problems where the challenge is to fit shapes within a container to minimise waste. In this example, the container must be completely filled using the minimum amount of panels. When the panel is homogenous, such as with sheet metal, it is desirable to reuse the off cuts to cover irregular regions at other places, as this has the potential to reduce the total number of sheets required.
The multi-objective optimisation problem investigated in this research is a variant of the basic layout optimisation problem. These optimisation problems share a common characteristic of the application of scalar-based search methods on resolving layout optimisation tasks that involve vector objects. More specifically, the layout optimisation task in question is defined by the following input data:

1. A range of standard rectangular sheets is provided to choose from

2. A set of linear shapes of arbitrary outlines are to be cut out from the standard sheets

The first input data is a set of standard rectangular sheets, which come in a variety of lengths and widths. For a given length and width, the sheets of the same size may be available in either fixed or unlimited numbers. One key task of the optimiser is to nominate the length and width from the available choices. Furthermore, in the case where a fixed number sheet is chosen and exhausted before all pieces are placed, the optimiser must be able to make another selection from the remaining available standard sheets.

The second input data is a set of shapes that are to be individually cut from the standard sheets. The advantage of Building Information Modelling (Azhar, 2011) is that the geometric data of the building can automatically be extracted and utilised to create these shapes. By making the selection on a standard rectangular sheet length and width, the optimiser cuts the shapes into grids using the standard sheet as a template. Areas that are not completely covered by a standard sheet will constitute the arbitrary shapes input to the optimiser.

Figure 2 shows an example of the visual representation of the input data for the case where the shapes are to be cut and calculated by the optimiser. In this example, a single target area is to be covered by standards sheets with a single choice of shape. The target area has an outline which includes convex and concave corners. Furthermore it also has a single void area that is not to be covered by the sheet material. With this input data defined, the optimiser is ready to calculate a solution. 


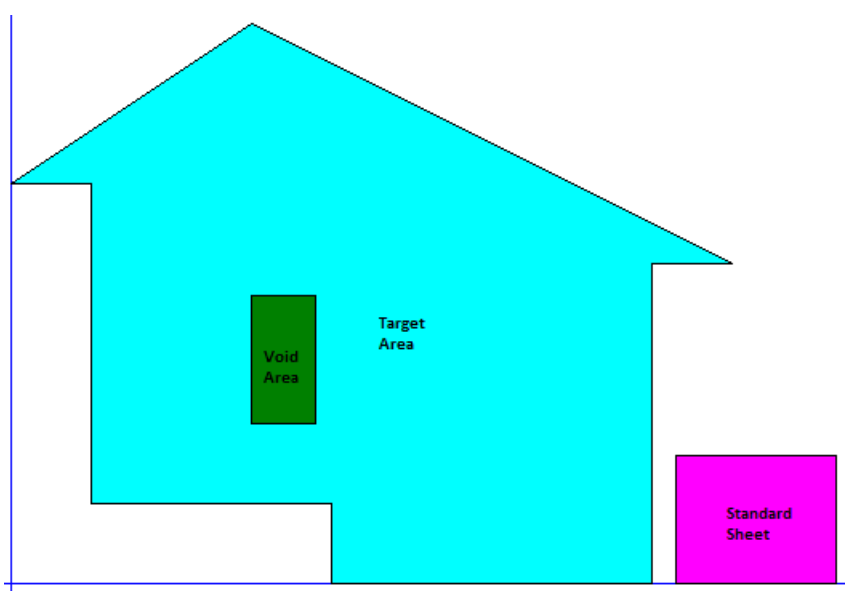

Figure 2. Layout optimisation problem

Figure 3 shows a typical sheet placement solution generated by the optimiser.

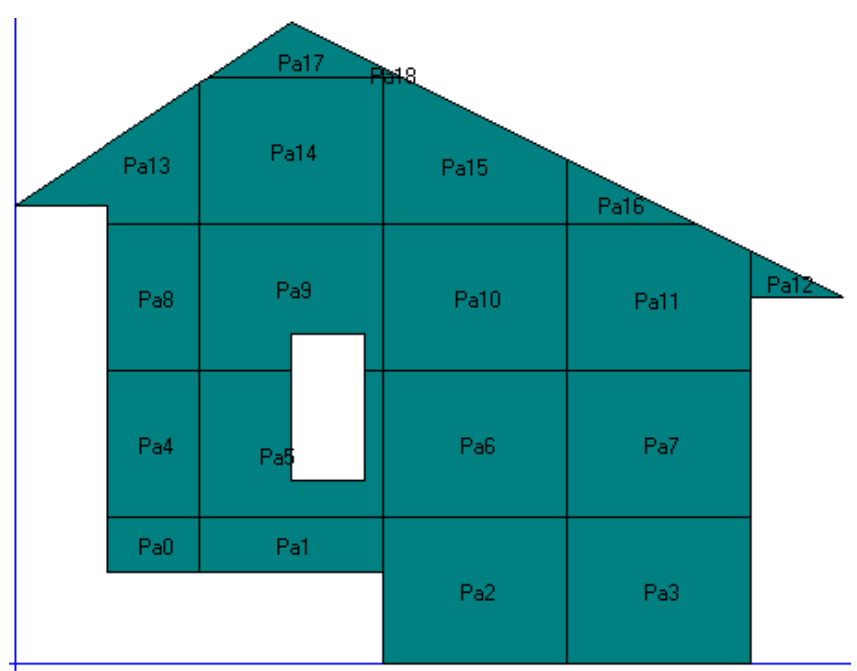

Figure 3. Sheet placement solution

Each piece covering the target area has been labelled with a unique panel number. There are 19 pieces in this solution, five of which have the size of a complete standard sheet $\left(\mathrm{Pa}_{2}\right.$, $\left.\mathrm{Pa}_{3}, \mathrm{~Pa}_{6}, \mathrm{~Pa}_{7} \& \mathrm{~Pa}^{10}\right)$. Whereas the rest occupy less space than a standard sheet. Figure 4 presents a cutting solution for the problem. The pieces that do not fit a complete standard are placed together to minimise waste. Note there is a gap required between parallel edges of adjacent pieces (e.g. between Pa15 and Pa16), which the optimiser accepts as an input parameter. Due to this gap, pieces that seemingly able to fit in a single sheet (such as $\mathrm{Pa} 1$ and $\mathrm{Pa} 8$ ) are placed in different sheets.

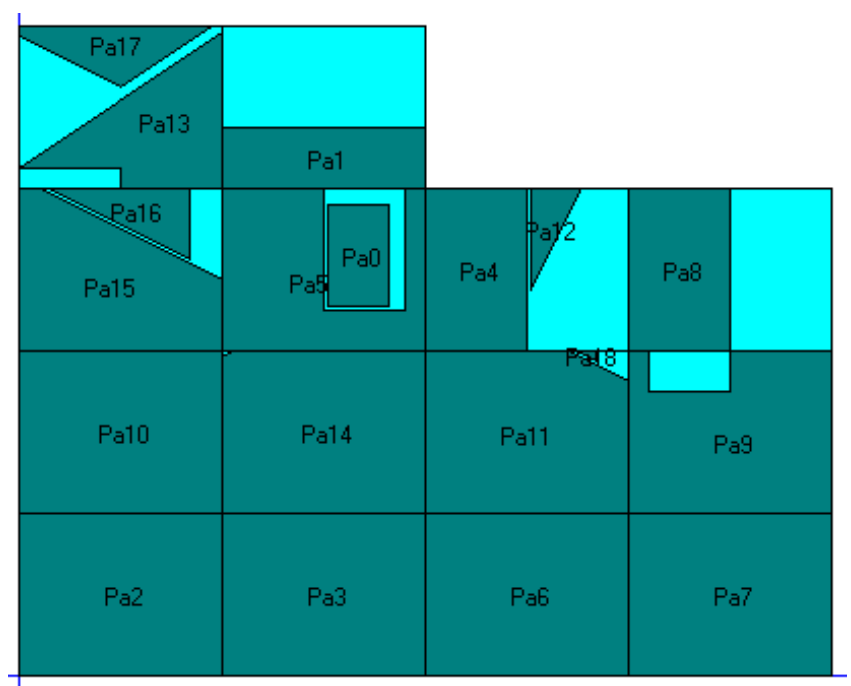

Figure 4. Layout cutting solution

Previous work has utilised a metaheuristic algorithm (i.e. Genetic Algorithm) to solve a single-objective version of the layout optimisation problem described above (Connor \& Siringoringo, 2007; Siringoringo, Connor, Clements, \& Alexander, 2008). In this wok, the optimisation task was divided into two distinct sub-tasks. The first is to find the "optimum" selection of standard sheet and its grid cutting arrangement. Whereas the second is to optimise the placement of the cut out shapes as a standard bin packing problem. Also the optimisation was a single-objective variant, where the only objective was the minimisation of waste.

The first sub-task was completed using a Genetic Algorithm. During the course of the research it was discovered that direct coding of the input parameters, such as the pieces translation and rotation, was not possible. There were two reasons direct coding scheme proved unsuitable for this class of problems. First, coding pieces translation and rotation information directly to the metaheuristic algorithm leads to the creation of large numbers of invalid solutions due to the violation of the no-overlap rule. Second, the information of which standard panel each piece should be mapped to cannot be effectively represented in the GA chromosomes. To address these issues, the concept of clusters was used.

In the cluster-based solution, the GA chromosome is represented as an array of integers. Each element of the array represents a piece to be nested in the standard sheet. The integer value held by the element refers to the number of a logical cluster where the piece belongs to. The evaluation function groups the elements to the designated cluster, and attempts to put pieces corresponding to a cluster together in a single standard sheet using a deterministic method. A special provision was made to reallocate pieces that can no longer placed with others of the same cluster to other sheets.

While the previous attempt succeeded in constructing a workable solution, it also has a few issues which restricts its 
use to the specific problem at hand, and prevents its application in a wider domain. For instance, the GA implementation was tightly integrated to the rest of the optimisation code, it is difficult to reuse the GA logic elsewhere. Similarly, it is difficult to replace the GA component of the solution with any other metaheuristic methods.

The second issue was the difficulty in adapting the optimiser to multi-objective version, since the GA logic was controlled by a higher level process. Therefore, modifying the optimiser to become multi-objective requires the modification of the GA as well as its controlling process, with the associated refactoring of the parameters passed between the software components.

The solution to these problems proposed in this paper are based on a model where the metaheuristic search controls the rest of the optimisation process. The optimisation problem is no longer broken into two separate tasks, the layout placement routines are configured so they serve as an evaluation function to the controlling metaheuristic search.

The basic premise of this solution is that pieces are placed on standard sheets sequentially, with the optimiser allocating new sheets as needed. Optimisation is achieved through seeking the placement sequence which produces the best results. This way, the optimisation task is reduced to a combinatorial problem from the metaheuristic search perspective. The actual placement of the pieces is delegated to a deterministic method. This ensures the evaluation function calculates exactly the same result for the same input parameters. For the purpose of this research, the deterministic method uses a naïve "best fit" strategy in order to produce good layout solutions. Such strategy simply seeks to place the pieces together where waste is minimised, regardless to what objective parameters the higher level optimiser uses. However, there is an open opportunity for further improve the performance of the deterministic method.

Reducing the search task to combinatorial problem makes it possible to use standards solutions to the classical Travelling Salesman Problem (TSP) and facilitates the ease of trialling different metaheuristics approaches without having to modify the problem representation. However, the model used does not fit the pure definition of TSP, because the value of an input sequence can only be calculated as a whole. Unlike in a standard TSP problem, in the layout optimisation the distance between nodes has no meaning, and hence no value can be purposefully assigned.

However the problem is still representable by a fully connected graph $\mathrm{G}$ made by vertices $\mathrm{V}$ and edges $\mathrm{E}$.

$\mathrm{G}=(\mathrm{V}, \mathrm{E})$

Since the distance between nodes is meaningless, for each edge in $\mathrm{E}$, there is no associated parameter $\mathrm{C}_{\mathrm{ij}}$ to expresses the cost of traversing the edge e $\in$ E which connects node $i \in V$ and node $\mathrm{j} \in \mathrm{V}$. Only the total cost can be calculated at for the complete tour, through an embodied objective function. The absence of information on the traversing cost for individual edges does have an adverse impact on any algorithm that calculates partial cost when constructing a tour, such as the Ant Colony Optimisation (ACO) method. Such methods can be further modified to take this into account (Siringoringo, 2016).

\section{Case Problem}

As discussed earlier, a typical surface tile optimisation problem calls for the complete covering of a collection of arbitrary shaped areas by same-sized panels arranged in contiguous rows and columns. Parts of the surface area that do not occupy whole panels, called the off cuts, are to be laid out together in stock panels of the same standard size as densely as possible. A collection of stock panels of varying dimensions is provided to complete the input. One of the optimiser key tasks is to make the selection of one stock panel, which is to be applied consistently for the entire solution. In maintaining simplicity of the design and the practicality of its application, the mixing of different panel dimensions within a single solution is not to be considered, although this could be addressed in future work.

The placement of offcut objects is further restricted by normal two-dimensional layout optimisation problem constraints. Firstly all of the objects must be completely enclosed by the plates. Secondly none of the objects can overlap.

The particular problem of this class under consideration is the placing of dry wall panels on the interior of a residential dwelling. A typical single storey dwelling with four bedrooms has been used in this work, and a view of the dwelling layout is given in Figure 5.

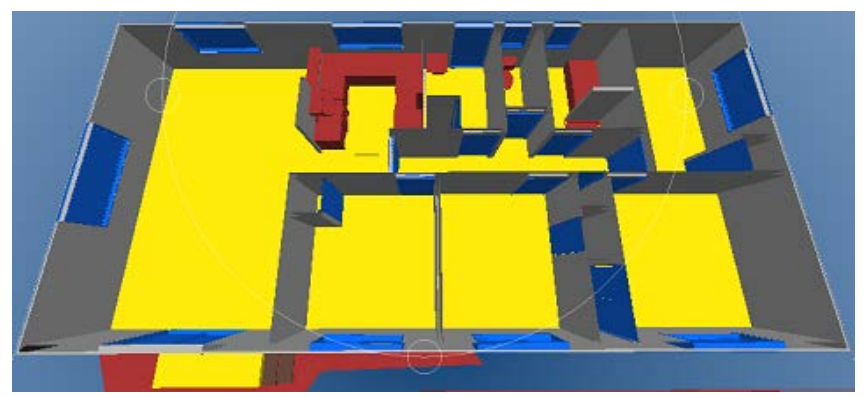

Figure 5. Residential dwelling

The output of the optimisation comes in two forms: a visual presentation of the layout plan and a set of scalar parameters that indicate the quality of the solution. The graphical information is to be used at the construction site to perform the physical cutting and placement, whereas the scalar data is used by downstream processes in the company's information system. 
Closer examination reveals that this problem is composed of two subproblems which must be resolved sequentially, although each sub-problem still belongs to the same twodimensional layout optimization. For a given enclosed area and a given dimensions of rectangular panels, the requirement is twofold:

1. Find the optimum arrangement of whole panels in which the covered area within the enclosure is maximized. The by-product of this process is a set of irregular shapes which represent the remaining exposed areas.

2. Resolve how such irregular shapes can be nested within the minimum number of panels. Shapes that are bigger than the panel itself are cut at angles parallel with the rectangle's axes to allow such nesting.

At the end of the calculation process, the desired output consists of numerical and graphical information:

1. The total number of panels, consisting of panels to be fitted whole and the remainder to be cut to produce the irregular shapes.

2. The nesting plan with which irregular shapes are cut from whole panels.

3. The area overlay plan with which whole panels and irregular cuts are fitted to the enclosed area.

It is important to note that although the two sub-problems are similar, they are resolved with mutually unrelated and potentially conflicting objectives. As an example, the lowest cost for the first sub-problem may be to cover as much area as possible with the least number of panels. However, the optimum solution to the second sub-problem may be the least amount of cutting. Hence a cheap solution in the first phase may lead to expensive penalties in the second.

\section{Results}

The geometry input data consists of the outline of the wall sections and a range of choices for the standard panel dimensions. No matching operations are required for the standard panel thickness or material grade. There are 13 standard panels available to choose from. Because the standard panels are always rectangular, the same set of panels reoriented by 90 degrees are also added to make the total number of choices of 26 . The small data set consists of wall sections from all the bedrooms, counting to 20 in total. Whereas the large data set consists of the 35 wall sections from the living room, the hallway, the kitchen, and all the bedrooms added together. A section of the extracted geometry is shown in Figure 6, which shows a single room only.

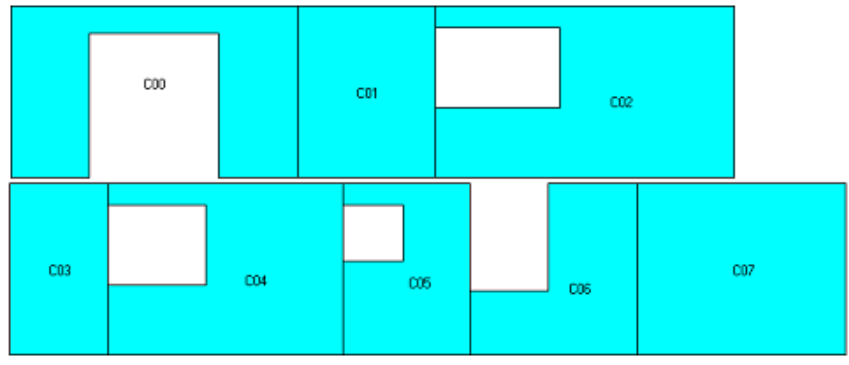

Figure 6. Living room wall sections

For the optimisation objective parameters, there two output variables to generate at each evaluation:

\section{Scrap Area (Minimisation)}

\section{Shared Edge Length (Maximisation)}

The material used on tile layout problems, such as plasterboard, generates off-cuts that are typically of negligible economical value. As such, no secondary computation is performed to reclaim unused o cut material, which is simply considered scrap. Minimising the total area of scrap material is the first objective of the optimisation.

Shared edge length is the total amount of cutting that results in creating new edges for two adjacent pieces. This is a maximisation parameter, where higher values mean less time and effort to carry out the physical act of cutting. Maximisation of parameters complicates the metaheuristic search's compare operations, making it necessary to reverse the parameter into a minimisation type. Shared edge is inverted to become a minimisation parameter by subtracting the value from a theoretical upper limit. However, there is no definite way to define the upper bound value prior to the optimisation. Because the shared edge length is a parameter dependent on other parameters, such as the total edge length of all the pieces, which is yet another output variable.

This problem is solved using the empirical approach of recording the actual values for this parameter during a number of pilot runs. It became apparent that the value ranges from 576.08 to 696.58 on the data set. Because the values fall within relatively small bands for each cases, it was decided that an artificial ceilings can be created by adding the width to the highest known value of the range. At the end of the actual experiment runs, it was discovered that the value ranges are 556.38 to 706.96 .

A number of metaheuristic algorithms have been executed to solve a common single-house dry wall layout optimisation problem. For comparison purposes, a random walk search has also been executed, which allows the performance of the respective algorithms to be investigated based on performance in the pareto-optimal space utilising a number of measures. To ensure statistically reliable results, each algorithm is executed against the input data multiple times, 
in this case for a total of 20 repetitions and the following results are based on the average performance across these repetitions.

Figure 7 presents the average performance of the algorithms on the spacing of solutions in the Pareto Front. The performance plot of the Random Walk is clearly separated from all other algorithms throughout the optimisation process. Whereas there are variations between the three metaheuristic algorithms at the initial generations, which become less significant as the search progresses.

\section{Figure 7. Convergence (Spacing)}

Despite the fluctuations, the Random Walk stays at higher spacing value compared to the other algorithms throughout the search. In contrast, the metaheuristic algorithms converge towards lower values with roughly the same pattern. Since lower spacing value is desirable, all the metaheuristic algorithms perform better than the Random Walk performs on this metric.

The difference of the convergence pattern of the Random Walk can be explained by the absence of pressure towards promising solutions. As a result, non-dominated solutions are discovered randomly scattered along the Pareto set. Whereas the metaheuristic algorithms purposefully narrow the search process to evaluate more possible candidates for every objective, yielding more evenly spaced solutions.

Figure 8 presents the average performance of the algorithms on the generational distance of solutions in the Pareto Front. All algorithms start at similar value range, except the Tabu Search which starts at significantly higher value, from which they continuously converge at a varying rate.
Figure 8. Convergence (Generational Distance)

The Random Walk search descends at a consistently lower gradient, and slows down the convergence earlier than the metaheuristic algorithms. Whereas the rest of the algorithms converge at similar rate, with the Tabu Search and Genetic Algorithm showing the most productive pattern.

The convergence patterns show that the Random Walk search is markedly less effective in moving the Pareto Front towards better solutions. Whilst the Tabu Search and Genetic Algorihtm perform best amongst the metaheuristic algorithms.

Figure 8 presents the average performance of the algorithms on the inverted generational distance of solutions in the Pareto Front. The performance plot of the Random Walk is clearly separated from all other algorithms throughout the optimisation process. Whereas there is relatively little variation between the three metaheuristic algorithms.

Figure 8. Convergence (Inverted Generational Distance)

The convergence plot of the algorithms follow a strongly similar pattern, with that of the Random Walk starting and staying at a consistent level higher than the other algorithms. Such patterns show that the Random Walk creates Pareto Fronts that are consistently dominated by those created by the metaheuristic algorithms. Whereas there is no appreciable difference among the metaheuristic algorithms on this aspect of performance.

Figure 9 presents the average performance of the algorithms on the hyper volume of solutions in the Pareto Front. The initial values are varied among the algorithms, with the Ant 
Colony and Genetic Algorithm scoring the highest, whereas Tabu Search scores the lowest.

Figure 9. Convergence (Hypervolume)

Beyond the initial few generations, the performance plot of the Random Walk falls below all other algorithms throughout the optimisation process. In contrast, the three metaheuristic algorithms remain grouped together, with the Tabu Search and Genetic Algorithm achieving a slightly better result than the Ant Colony at the end of the search.

Figure 10 presents the average contributions of the algorithms to the final set of solutions in the Pareto Front. All algorithms start with the same initial value of zero, from which the metaheuristic algorithms raise at markedly different rates.

Figure 10. Convergence (Contribution)

The Tabu Search contributes most and early, with the Genetic Algorithm following at significantly lower numbers later, and the Ant Colony contributing only marginally at the last few generations of the search. Whereas the Random Walk remains at zero throughout the process. Such results indicate that the metaheuristic algorithms are able to contribute nondominated solutions to the aggregated Pareto Front at every generation, with the Tabu Search being the most productive and the Ant Colony the least. The Random Walk on the other hand, fails to contribute non-dominated solutions during the entire search.

Figure 11 shows a set of the non-dominated solutions in the normalised objective space.
Figure 11. Pareto-optimal solutions

The actual objective values of a selected few of these solutions are presented in Table 1 , which shows the five candidates where the Euclidean distance to the origin is the least.

\begin{tabular}{ccc}
\hline & $\begin{array}{c}\text { Scrap Area (m2) } \\
\text { [Minimise] }\end{array}$ & $\begin{array}{c}\text { Shared Edges (m) } \\
\text { [Maximise] }\end{array}$ \\
\hline $\mathbf{1}$ & 14.490 & 474.489 \\
\hline $\mathbf{2}$ & 16.166 & 488.882 \\
\hline $\mathbf{3}$ & 17.734 & 512.118 \\
\hline $\mathbf{4}$ & 25.616 & 692.715 \\
\hline $\mathbf{5}$ & 38.216 & 699.166 \\
\hline
\end{tabular}

Table 1. Candidate solution objective values

The solutions presented illustrate the true power of the multiobjective optimisation approach as they offer a set of solutions from which the designer could select the most appropriate. For a dry wall material such as GIB board, the designer may choose to trade off higher material waste against a reduction in labour achieved through a higher shared edge. However, for a more expensive material the designer would likely choose an alternative solution from the pareto-optimal set.

There are two objective values to be measured. The material waste presents the amount of tiling material that cannot be reused and have to be discarded. Whereas the shared edge presents the length of the pieces that overlap with either the plate's boundary or other pieces' edges, indicating the effort required to perform the actual cutting.

\section{Discussion}

Analysing the results given in Section 5, it can be seen that there is a consistent pattern where the metaheuristic algorithms perform well, in comparison to the Random Walk. The metaheuristic algorithms perform particularly better than the Random Walk on the spacing convergence aspect. Which 
is consistent with its unbiased search pattern that does not necessarily produce evenly spread non-dominated solutions.

The metaheuristic algorithms on the other hand, focus the search on specific areas only. This results in better nondominated solutions that are spread more evenly. For all other convergence measurements, the metaheuristic searches also perform better than the Random Walk. Although the discrepancy between these algorithms are generally small, suggesting that the representation of the problem is allowing different algorithms to perform to their full potential.

The Genetic Algorithm and the Tabu Search perform at very similar levels where both achieve the best performance on certain metrics, with the Ant Colony following closely behind. On the Contribution aspect however, the Tabu Search performs well above others, contributing more nondominated solutions to the aggregate Pareto Front than others.

The general observation from this experiment is that metaheuristic algorithms converge well on all aspects directly related to the quality of the non-dominated solutions. Which indicates that the metaheuristic algorithms respond well to the novel presentation of the geometry problem, and able to efficiently converge towards optimum solution set in a similar fashion they do on scalar problems.

\section{Conclusion}

This research in this paper has investigated the application of three different metaheuristic search algorithms to a layout optimisation problem typical of residential construction, namely the design of a drywall layout. This optimisation has been undertaken to both minimise the waste and optimise the cutting layout as a means to minimise the time and effort to carry out the physical act of cutting. All three algorithms investigated have the potential to explore the solution space and find candidate solutions that effectively trade-off these two conflicting objectives.

\section{References}

Adamowicz, M., \& Albano, A. (1976). Nesting twodimensional shapes in rectangular modules. Computer-Aided Design, 8(1), 27-33.

Azhar, S. (2011). Building information modeling (BIM): Trends, benefits, risks, and challenges for the AEC industry. Leadership and management in engineering, 11(3), 241-252.

Connor, A. M., \& Siringoringo, W. S. (2007, 7-9 November). Using genetic algorithms to solve layout optimisation problems in residential building construction. Paper presented at the 20th International Conference on Computer Applications in Industry and Engineering, San Francisco, USA.

Coté, J.-F., Gendreau, M., \& Potvin, J.-Y. (2014). An exact algorithm for the two-dimensional orthogonal packing problem with unloading constraints. Operations Research, 62(5), 1126-1141.
Cranny, T. R., Bossomaier, T., \& Sibley-Punnett, L. (1999). Optimisation of layout for a problem in roofing with sheets. Paper presented at the 1999 Congress on Evolutionary Computation, Washington, DC.

Crossin, E., Hedayati, M., \& Clune, S. (2014). Waste avoidance and reuse strategies for residential buildings in Australia. Retrieved from

Dyckhoff, H. (1990). A typology of cutting and packing problems. European Journal of Operational Research, 44(2), 145-159.

Echenagucia, T. M., Capozzoli, A., Cascone, Y., \& Sassone, M. (2015). The early design stage of a building envelope: Multi-objective search through heating, cooling and lighting energy performance analysis. Applied Energy, 154, 577-591.

Epstein, L., Imreh, C., \& Levin, A. (2010). Class constrained bin packing revisited. Theoretical Computer Science, 411(34-36), 3073-3089.

Georgis, N., Petrou, M., \& Kittler, J. (2000). On the generalised stock-cutting problem. Machine Vision and Applications, 11(5), 231-241.

Harren, R., \& Kern, W. (2011). Improved lower bound for online strip packing. Paper presented at the 9th Workshop on Approximation and Online Algorithms, Saarbruecken, Germany.

Hifi, M. (1998). Exact algorithms for the guillotine strip cutting/packing problem. Computers \& Operations Research, 25(11), 925-940.

Hong, W.-K., Lee, G., Lee, S., \& Kim, S. (2014). Algorithms for in-situ production layout of composite precast concrete members. Automation in Construction, 41, 50-59.

Labbé, M., Laporte, G., \& Martello, S. (1995). An exact algorithm for the dual bin packing problem. Operations Research Letters, 17(1), 9-18.

Lodi, A., Martello, S., \& Vigo, D. (2004). Models and bounds for two-dimensional level packing problems. Journal of Combinatorial Optimization, 8, 363-379.

Nimtawat, A., \& Nanakorn, P. (2010). A genetic algorithm for beam-slab layout design of rectilinear floors. Engineering Structures, 32(11), 3488-3500.

Rao, C. S., Geevarghese, J. J., \& Rajan, K. (2010). Improved approximation bounds for vector bin packing. arXiv preprint arXiv:1007.1345.

Shigehiro, Y., Koshiyama, S., \& Masuda, T. (2005). A new approach to rectangle packing problem based on stochastic tabu search. Transactions of the Society of Instrument and Control Engineers, 40(7), 747754.

Sibley-Punnet, L., \& Bossomaier, T. (2001). Optimisation techniques for roof layout. Paper presented at the IEEE Region 10 International Conference on Electrical and Electronic Technology, Phuket Island, Thailand.

Siringoringo, W. (2016). Exploration of a Hybrid MultiObjective Heuristic Architecture for Solving 2D Layout Problems. (PhD (Unpublished)), Auckland University of Technology.

Siringoringo, W., Connor, A. M., Clements, N., \& Alexander, N. (2008). Minimum cost polygon overlay with rectangular shape stock panels. 
International Journal of Construction Education and Research, 4(3), 153-176.

Snudden, J., Ward, C., \& Potter, K. (2014). Reusing automotive composites production waste. Reinforced Plastics, 58(6), 20-27.

Tokuyama, H., \& Ueno, N. (1985). The cutting stock problem for large sections in the iron and steel industries. European Journal of Operational Research, 22(3), 280-292.

Vassiliadis, V. S. (2005). Two-dimensional stock cutting and rectangle packing: binary tree model representation for local search optimization methods. Journal of Food Engineering, 70(3), 247-268.

Wang, N., \& Adeli, H. (2014). Sustainable building design. Journal of Civil Engineering and Management, 20(1), 1-10.

Wascher, G., Heike, H., \& Schumann, H. (2007). An improved typology of cutting and packing problems. European Journal of Operational Research, 183(3), 1109-1130.

Yang, M.-D., Lin, M.-D., Lin, Y.-H., \& Tsai, K.-T. (2017). Multiobjective optimization design of green building envelope material using a non-dominated sorting genetic algorithm. Applied Thermal Engineering, 111, 1255-1264.

Zou, P., Hardy, R., \& Yang, R. (2015). Barriers to building and construction waste reduction, reuse and recycling: a case study of the Australian Capital Region. Paper presented at the Building Today Saving Tomorrow: Sustainability In Construction And Deconstruction Conference Auckland, New Zealand. 